\title{
miR-1247-5p functions as a tumor suppressor in human hepatocellular carcinoma by targeting Wnt3
}

\author{
YUANKUI CHU ${ }^{1,3^{*}}$, WEINING FAN ${ }^{3 *}$, WENWEI GUO $^{3 *}$, YIXIN ZHANG $^{3}$, LIXIN WANG $^{1}$, \\ LE GUO $^{1-3}$, XIANGGUO DUAN ${ }^{1,3}$, JUN WEI ${ }^{1,2}$ and GUANGXIAN XU ${ }^{1-3}$ \\ ${ }^{1}$ Medical Experimental Center, General Hospital of Ningxia Medical University; ${ }^{2}$ Ningxia Key Laboratory of Clinical \\ and Pathogenic Microbiology and ${ }^{3}$ Department of Laboratory Medicine, \\ General Hospital of Ningxia Medical University, \\ Yinchuan, Ningxia 750004, P.R. China
}

Received October 24, 2016; Accepted November 20, 2016

DOI: 10.3892/or.2017.5702

\begin{abstract}
Increasing evidence suggests that aberrant expression of certain microRNAs (miRNAs) may participate in the genesis and progression of tumors. Several studies have indicated that miR-1247-5p plays different roles in various types of cancer cells. The effects of miR-1247-5p on human hepatocellular carcinoma (HCC) cells are elusive. In the present study, we investigated the effects of miR-1247-5p on the progression of HCC. The transcript of miR-1247-5p was markedly downregulated in clinical samples of patients with $\mathrm{HCC}$ and HCC cell lines, and ectopic overexpression of miR-1247-5p markedly inhibited the proliferation and invasion of HepG2 cells, induced cell apoptosis in vitro, and suppressed the growth of transplanted tumors in vivo. Wnt3 was found to be a potential target of miR-1247-5p and overexpression of miR-1247-5p was able to significantly downregulate the expression of Wnt3 by directly targeting the 3'UTR of this gene, which was verified by luciferase reporter assay and western blotting. Furthermore, we found that the miR-1247-5p gene was hypermethylated in HepG2 cells, and the transcript of miR-1247-5p was increased significantly after treatment with the demethylation drug 5-azacytidine. These findings demonstrated that miR-1247-5p functions as a tumor suppressor in human HCC by targeting Wnt 3 and that the expression of miR-1247-5p can be regulated by DNA methylation, which indicates that miR-1247-5p has the potential to be a therapeutic target as well as a diagnostic marker of HCC.
\end{abstract}

Correspondence to: Dr Guangxian Xu, Department of Laboratory Medicine, General Hospital of Ningxia Medical University, Yinchuan, Ningxia 750004, P.R. China

E-mail: xuguangxian@nxmu.edu.cn

*Contributed equally

Key words: hepatocellular carcinoma, microRNA-1247-5p, Wnt3, DNA methylation

\section{Introduction}

Hepatocellular carcinoma (HCC) is a common malignant tumor of the digestive tract (1). The incidence of HCC has increased significantly in recent years, and the overall survival of patients with HCC remains unsatisfactory. Similar to other malignancies, the development of HCC is a long-term, multistep process characterized by the alteration of genes. However, the molecular mechanisms involved in the initiation and progression of HCC are still poorly understood. Recently, the role of epigenetic regulation, particularly DNA methylation and the aberrant expression of miRNAs, in the occurrence and development of cancers is gradually being recognized $(2,3)$.

MicroRNAs (miRNAs) are single stranded, small noncoding RNAs of 18-25 nucleotides in length. They can negatively regulate gene expression through base-pairing to the $3^{\prime}$ untranslated region (3'UTR) of target mRNAs, resulting in translation inhibition or mRNA degradation (4-6). It is currently estimated that $30 \%$ of coding genes in humans are regulated by miRNAs (7). More and more studies suggest that beyond involvement in various biological processes, including cell growth, differentiation and apoptosis (8-10), dysregulation or dysfunction of miRNAs contributes to the genesis and progression of cancer. Several studies have revealed that miRNAs participate in the initiation and progression of HCC. miRNA-375 inhibited the invasion and differentiation of HCC cells (11), and miRNA-199a-3p induced G1 phase arrest and promoted the efficacy of doxorubicin in HCC cells (12). It is expected that by exploring the role of miRNAs in tumorigenesis and progression a new approach for the early diagnosis and treatment of HCC may be provided.

miR-1247-5p, located at the distal end of human chromosome 14 (13) and conservatively expressed in mammalian species, was found to be differentially expressed in cartilage (14) as well as in breast (15), colorectal (16) pancreatic (17), and prostatic cancer cells (18), and had different effects on proliferation and invasion in various cancer cells. Currently, one study reported that the miR-1247-5p gene was hypermethylated in clinical samples of patients with HCC (19). However, the expression level and functional effects of miR-1247-5p in HCC are largely unknown. 
In the present study, we demonstrated a significant downregulation of miR-1247-5p in the clinical samples of HCC patients and HCC cell lines. Ectopic overexpression of miR-1247-5p inhibited the proliferation and invasion of HepG2 cells, induced cell apoptosis in vitro, and suppressed the growth of transplanted tumors in vivo. Notably, we demonstrated that miR-1247-5p directly targeted the 3'UTR of the Wnt3 gene, and that the expression of miR-1247-5p could be regulated by DNA methylation. Our findings provide valuable evidence elucidating the function and regulatory mechanisms of miR-1247-5p in human HCC, and indicate that miR-1247-5p can be used as a potential therapeutic target as well as a diagnostic marker of HCC.

\section{Materials and methods}

Patients and tissue specimens. Paraffin-embedded samples, including $16 \mathrm{HCC}$ tumor tissues and 10 non-tumor tissues, as well as serum samples from 41 HCC patients and 41 healthy volunteers were obtained at the General Hospital of Ningxia Medical University (Yinchuan, Ningxia, China). All of the tumor samples were confirmed by pathologists and none of the patients had undergone any therapy before recruitment to this study. The use of clinical material for all experiments was approved by the Ethics Committee of the General Hospital of Ningxia Medical University.

Cell lines and cell culture. The human HCC cell lines, HepG2, HCCLM3 and SMMC-7721, and normal liver cell line LO2 were purchased from Shanghai Fu Meng Biotechnology Inc. (Shanghai, China). HEK-293T cells were stored at our laboratory. HepG2, HCCLM3 and SMMC-7721 cells were cultured in Dulbecco's modified Eagle's medium (DMEM), while LO2 cells were cultured with RPMI-1640 medium, containing $10 \%$ heat-inactivated fetal bovine serum (FBS) (both from Gibco/Life Technologies, Grand Island, NY, USA), 100 U/ml penicillin, $100 \mu \mathrm{g} / \mathrm{ml}$ streptomycin, $15 \mathrm{mmol} / 1 \mathrm{HEPES}$ and $200 \mathrm{mmol} / \mathrm{l} \mathrm{L}$-glutamine. All of the cells were incubated at $37^{\circ} \mathrm{C}$ in a humidified incubator containing $5 \% \mathrm{CO}_{2}$.

Quantitative reverse transcriptase-polymerase chain reaction ( $q R T-P C R)$. Total RNA was extracted using an RNA extraction kit (Omega Bio-Tek, Inc., Norcross, GA, USA) from paraffin-embedded tissues. An miRNA purification kit (Kangweishiji Biotech Co., Ltd., Beijing, China) was used for serum samples and TRIzol reagent (Invitrogen, Carlsbad, CA, USA) was used for cells according to the manufacturer's protocol. Then, $1 \mu \mathrm{g}$ of total RNA from each sample was reverse transcribed to single-stranded cDNA with EasyScript First-Strand cDNA Synthesis SuperMix (TransGen Biotech Co., Ltd., Beijing, China) and the miR-1247-5p stem-loop RT primer sequence was: 5'-CTCAACTGGTGTCGTGGAGT CGGCAATTCAGTTGAGTCCGGGGAC-3'. The mRNA expression of miR-1247-5p was quantified with the TransStart Top Green qPCR SuperMix (TransGen Biotech Co., Ltd.) and the StepOnePlus qPCR system (ABI, Carlsbad, CA, USA) under the following conditions: $95^{\circ} \mathrm{C}$ for $5 \mathrm{~min}$ followed by 40 cycles consisting of $95^{\circ} \mathrm{C}$ for $15 \mathrm{sec}, 60^{\circ} \mathrm{C}$ for $15 \mathrm{sec}$ and $72^{\circ} \mathrm{C}$ for $15 \mathrm{sec}$. The relative gene expression levels of miR-1247-5p were calculated using the $2^{-\Delta \Delta \mathrm{Ct}}$ method, and U6 snRNA was used for normalization. The oligonucleotide sequences of the primers are shown in Table I.

Plasmid construction and transfection. Oligonucleotide sequences of miR-1247-5p precursor (MIMAT0005899, miRBase) were designed using the Ambion Company online software: forward sequence, 5'-GTT^AACACCCGTCCCGCT TGTCCCCGGATTCAAGAGATCCGGGGACGAACGGG ACGGGTTTTTTTC ${ }^{\wedge}$ TCGAG-3' $^{\prime}$ and reverse sequence, 5'-CA A $^{\wedge}$ TTGTGGGCAGGGCAAGCAGGGGCCTAAGTTCTTC TAGGCCCCTGCTTGCCCTGCCCAAAAAAAGAGCT^C-3', and synthesized by Sangon Biotech Inc. (Shanghai, China). After annealing, miR-1247-5p precursor was subcloned between $H p a \mathrm{I}-X h o \mathrm{I}$ restriction sites in lentiviral vector $\mathrm{pSicoR}$ to generate pSicoR-miR-1247-5p (LV-miR-1247-5P) and pSicoR empty vector was used as a negative control (LV-NC). The miR-1247-5p inhibitor (Inh-miR-1247-5p) and its negative control (Inh-NC) were purchased from GenePharma Inc. (Shanghai, China). The 3'UTR and the mutant 3'UTR of the Wnt3 gene were amplified (primer sequences are shown in Table I) and subcloned into the pMIR-Luc reporter plasmid (Promega, Madison, WI, USA) between SpeI-HindIII to generate pMIR-Luc-Wnt3 and pMIR-Luc-mut-Wnt3 recombinant plasmid. All of the constructs were verified by sequencing.

The recombinant lentiviral vectors pSicoR-miR-1247-5p were co-transfected with pCMV-VSV-G and pCMV-dR 8.91 into HEK-293T cells using Lipofectamine 3000 (Invitrogen) according to the manufacturer's instructions. After $48 \mathrm{~h}$ of incubation, the supernatant of the cultures was collected and concentrated and then used to infect HepG2 cells. The pSicoR empty vector was used as a negative control using the same protocol. The miR-1247-5p inhibitor and its negative control, pMIR-Luc-Wnt3 and pMIR-Luc-mut-Wnt3 recombinant plasmid were transfected into HepG2 cells using Lipofectamine 3000 for $48 \mathrm{~h}$.

Cell proliferation assay. Cell proliferation activity was detected using the TransDetect Cell Counting Kit-8 (CCK-8) (TransGen Biotech Co., Ltd.). In brief, HepG2 cells were seeded at $5 \times 10^{3}$ cells/well in 96 -well plates. At various time-points, $10 \mu \mathrm{l}$ of CCK-8 solution was added to each well and incubated for another $2 \mathrm{~h}$ at $37^{\circ} \mathrm{C}$. The absorbance was assessed at a wavelength of $450 \mathrm{~nm}$ using the MK3 ELISA reader (Thermo Fisher Scientific, Inc., Waltham, MA, USA).

Cell invasion assay. Transwell assay was performed to analyze the role of miR-1247-5p in the invasive activity of HepG2 cells. Briefly, cells were trypsinized and added to the upper Transwell chambers coated with Matrigel (BD, Franklin Lakes, NJ, USA). The lower chambers were filled with fresh medium containing $15 \%$ FBS. After a 48-h incubation, the cells on the upper surface were removed and the cells on the lower surface were fixed in $4 \%$ formaldehyde (Sigma-Aldrich, St. Louis, MI, USA). Then, the fixed cells were stained with $0.1 \%$ crystal violet (Sigma-Aldrich). Subsequently, the cells in each group were photographed in five randomly selected fields using an Olympus IX71 microscope (Olympus, Shinjuku-ku, Tokyo, Japan). The number of invading cells was calculated and analyzed with GraphPad Prism 6 (Graph Pad Software, La Jolla, CA, USA). 
Table I. Oligonucleotide sequences.

Name

Sequence (5' to $3^{\prime}$ )

\section{miR-1247-5p-F}

U6-F

U6-R

Wnt3-utr-F and Wnt3-mut-F

Wnt3-utr-R

Wnt3-mut-R

Methylation-specific primer-F

Methylation-specific primer-R

Unmethylation primer-F

Unmethylation primer-R
ACACTCCAGCTGGGACCC

CTCGCTTCGGCAGCACA

AACGCTTCACGAATTTGCGT

CCCAAGCTTGGGGGATTCAGCGAAGTCTCA

GACTAGTCAAGCCTCAGGTCTGTTCC

GACTAGTCTATTGTCACAGGCGAGTTGGGTCTGG

AGGGAGTTGTTTCGTATTTTTAAAC

GAACGTTACTCTCTACCCCGAA

GGAGTTGTTTTGTATTTTTAAATGT

CAAACATTACTCTCTACCCCAAA

F, forward; R, reverse.

Cell cycle assay. The cell cycle was analyzed using flow cytometry. In brief, the cells were seeded at $1 \times 10^{4}$ cells $/ \mathrm{cm}^{2}$ in 6 -well plates and incubated for $72 \mathrm{~h}$. Then, the cells were harvested by trypsinization, washed in ice-cold phosphate-buffered saline (PBS), and fixed in $75 \%$ ethanol at $4^{\circ} \mathrm{C}$ overnight. Subsequently, the cells were rinsed twice in chilled PBS, incubated with $2 \mu \mathrm{g} / \mathrm{ml}$ of RNAase at $37^{\circ} \mathrm{C}$ for $30 \mathrm{~min}$ and then rinsed twice and incubated with $20 \mu \mathrm{g} / \mathrm{ml}$ of propidium iodide (Beyotime Biotech, Beijing, China) at room temperature for $1 \mathrm{~h}$. Finally, the cells in each group were analyzed by flow cytometry (FACSCalibur; BD Biosciences, San Jose, CA, USA).

Hoechst staining assay. HepG2 cells were plated with $1 \times 10^{5}$ cells $/ \mathrm{ml}$ into 6 -well plates. Then, the cells were infected with lentiviral-mediated miR-1247-5p or its negative control for $48 \mathrm{~h}$, or transfected with inhibitors of miR-1247-5p or its negative control using Lipofectamine 3000 for 48 h. Subsequently, the cells in each group were stained with a Hoechst staining kit (Beyotime, Shanghai, China). The nuclear morphology was observed under an Olympus IX71 fluorescence microscope.

Tumorigenicity assay. Ten BALB/c nude mice, purchased from the Experimental Animal Center of Ningxia Medical University, were randomly divided into two groups. All mice were housed and maintained under specific pathogen-free conditions, and the use of mice for this study was approved by the Ethics Committee of the General Hospital of Nigxia Medical University. In the experimental group, the mice were subcutaneously injected with $1 \times 10^{7}$ HepG 2 cells infected with LV-miR-1247-5p at the lateral axillary. From the first day of injection, the tumor size was assessed once every two days for four weeks. Then, the mice were sacrificed and the tumors were photographed. The mice in the control group were injected with HepG2 cells infected with lentiviral empty vectors using the same protocol.

Luciferase assay. The specificity of miR-1247-5p targeting the 3'UTR of the Wnt3 gene was ascertained by co-transfection of plasmid DNA of pMIR-Luc-Wnt3 or pMIR-Luc-mut-Wnt3 recombinant plasmid with pSicoR-
miR-1247-5P (LV-miR-1247-5P) or pSicoR empty plasmid (LV-NC), inhibitor of miR-1247-5p (Inh-miR-1247-5p) or negative control of inhibitor (Inh-NC) into HepG2 cells, and determined by the relative firefly luciferase activity expressed in relative light units (RLU) at 48 h post-transfection using a Dual-Luciferase Reporter Assay kit (Promega). A Renilla luciferase expressing plasmid pRL-TK (Promega) was always included in the transfection to normalize the efficiency of each transfection.

Western blot analysis. Total proteins were extracted from cells using RIPA lysis buffer containing $1 \mathrm{X}$ protease inhibitor cocktail (Beyotime), and the protein concentration was calculated using the BCA protein assay kit (KeyGen Biotech Inc., Nanjing, China). Western blot analysis was carried out according to the standard protocol. Briefly, $40 \mu \mathrm{g}$ of proteins were separated by $10 \%$ SDS-PAGE gel electrophoresis and transferred to polyvinylidine fluoride (PVDF) membranes (Millipore, Bedford, MA, USA) using $95 \mathrm{~mA}$ at $4^{\circ} \mathrm{C}$ for $2 \mathrm{~h}$. After blocking in 5\% non-fat dry milk in Tris-buffered saline (TBS), the membranes were incubated with a rabbit polyclonal antibody against $\beta$-catenin (51067-2-AP), a rabbit polyclonal against GAPDH (10494-1-AP) (both from Proteintech, Rosemont, IL, USA), a goat polyclonal against Wnt3 (sc5213; Santa Cruz Biotechnology, Inc., Santa Cruz, CA, USA) and a rabbit polyclonal against $\beta$-actin (ab8227; Abcam, Cambridge, MA, USA) at 1:500 dilution in TBS overnight at $4^{\circ} \mathrm{C}$. The membranes were then washed three times with TBS-Tween-20, and subsequently incubated with secondary antibodies [anti-rabbit IgG-horseradish peroxidase-conjugated and anti-goat IgG-horseradish peroxidase-conjugated (ZSGB-Bio Inc., Beijing, China)], conjugated with horseradish peroxidase at a 1:1,000 dilution in TBS for $2 \mathrm{~h}$ at room temperature. The membranes were washed again in TBS-Tween-20 six times at room temperature. The protein bands were visualized using the Luminol reagent (Thermo Fisher Scientific, Inc.) and detected using an enhanced chemiluminescence detection system.

DNA methylation analysis. The sequence of the promoter region of the miR-1247-5p gene was analyzed using UCSC 
A

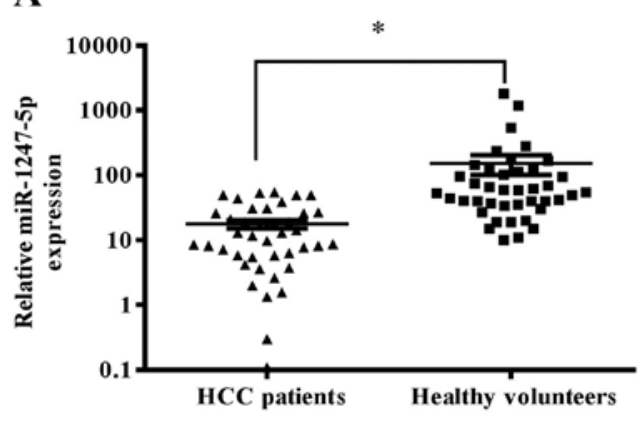

B

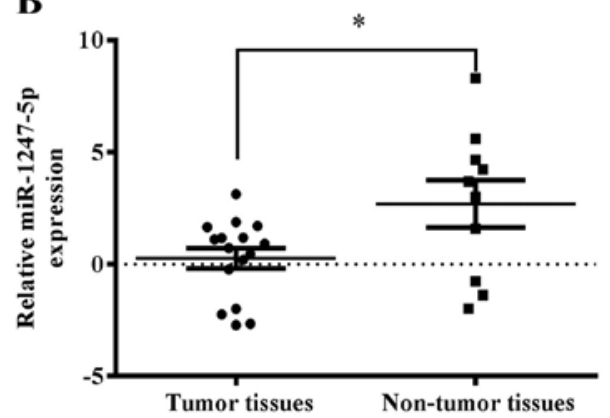

C

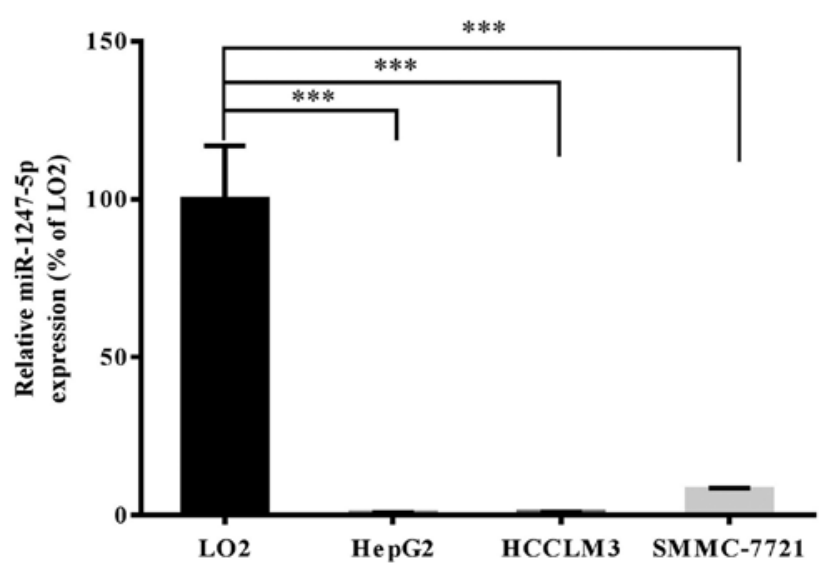

Figure 1. Expression of miR-1247-5p (qRT-PCR) in HCC clinical samples and cell lines. miR-1247-5p is downregulated in clinical samples of patients with HCC. Relative mRNA expression of miR-1247-5p in serum samples $(A, n=41)$ and tumor tissues $(B$, n=16) of patients with HCC. (C) miR-1247-5p is downregulated in HCC cell lines. Relative mRNA expression of miR-1247-5p in HCC cell lines HepG2, HCCLM3 and SMMC-7721 and normal liver cell line LO2. Graphs represent the relative expression values, normalized against the reference gene. Data are expressed as the mean $\pm \mathrm{SD} ;{ }^{*} \mathrm{p}<0.05,{ }^{*} *{ }^{*}<0.0005$. HCC, hepatocellular carcinoma.

Genome Database (http://genome.ucsc.edu/). Genomic DNA of HepG2 cells, and HepG2 cells treated with 5 or $10 \mu \mathrm{mol} / 1$ of 5-azacytidine for $48 \mathrm{~h}$ were extracted using a DNA extraction kit (Promega) and sulfated using the EpiTect Bisulfite kit (Qiagen, Schnackenburgallee, Hamburg, Germany) according to the manufacturer's instructions. Then, the bisulfite-modified DNA was used as a template for the amplification of DNA methylation-specific PCR (MSP). Normal liver cell line LO2 was used as a normal control. Methylation-specific primers and unmethylation primers were designed using MethPrimer online software and synthesized by GenePharma Inc. (primer sequences are shown in Table I). The reactions were incubated at $95^{\circ} \mathrm{C}$ for $5 \mathrm{~min}$, followed by 40 cycles of $95^{\circ} \mathrm{C}$ for $30 \mathrm{sec}$, $58^{\circ} \mathrm{C}$ for $30 \mathrm{sec}$ (methylation-specific primers) or $56^{\circ} \mathrm{C}$ for $30 \mathrm{sec}$ (unmethylation primers), $72^{\circ} \mathrm{C}$ for $30 \mathrm{sec}$, and then $72^{\circ} \mathrm{C}$ for $10 \mathrm{~min}$. PCR products were detected by $2.5 \%$ agarose gel electrophoresis, and photographed using a gel imaging system (Bio-Rad, Hercules, CA, USA).

Statistical analysis. All data collected in the present study, were obtained from at least three independent experiments for each condition. SPSS 19.0 analysis software (SPSS, Inc., Chicago, IL, USA) and GraphPad Prism 6 (Graph Pad Software, La Jolla, CA, USA) were used for the statistical analysis. Statistical evaluation of the data was performed by one-way ANOVA when more than two groups were compared with a single control, and t-test for comparison of differences between the two groups. Significant differences were assigned to p-values $<0.05,<0.01,<0.0005$ and $<0.0001$. Data are presented as the mean \pm standard deviation (SD).

\section{Results}

miR-1247-5p is downregulated in HCC. The mRNA expression of miR-1247-5p in clinical samples of HCC patients and HCC cell lines was analyzed by qRT-PCR. The results revealed that miR-1247-5p was significantly downregulated in tumor samples $(n=16)$ and serum samples $(n=41)$ of HCC patients compared to non-tumor samples $(n=10)$ and serum samples $(n=41)$ of healthy volunteers (Fig. 1A and B). Subsequently, the mRNA expression level of miR-1247-5p was assessed in HCC cell lines, HepG2, HCCLM3 and SMMC-7721, and normal liver cell line LO2. The results revealed that the mRNA expression of miR-1247-5p was significantly decreased in the HCC cell lines, HepG2 $(0.721 \pm 0.090 \%$; p<0.0005), HCCLM3 $(0.890 \pm 0.097 \% ; \mathrm{p}<0.0005)$ and SMMC-7721 $(8.185 \pm 0.355 \% ; \mathrm{p}<0.0005)$ normalized to the normal liver cell line LO2 (Fig. 1C). The HepG2 cells were chosen for the subsequent experiments due to the lowest expression of miR-1247-5p.

miR-1247-5p inhibits cell proliferation and invasion in vitro. The role of miR-1247-5p in the progression of HCC was investigated using CCK- 8 and Transwell assays. The mRNA expression of miR-1247-5p in HepG2 cells infected with lentiviral-mediated miR-1247-5p (LV-miR-1247-5p) or its 
A

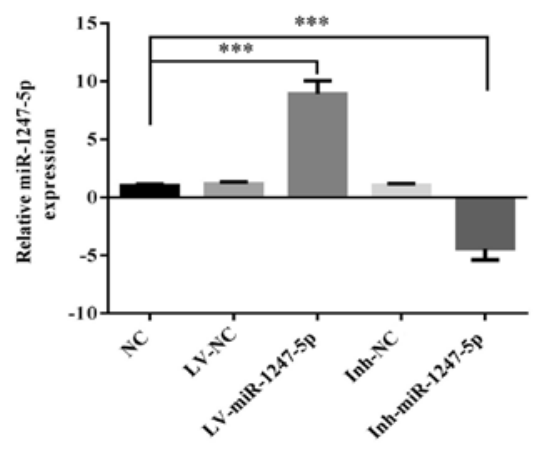

C

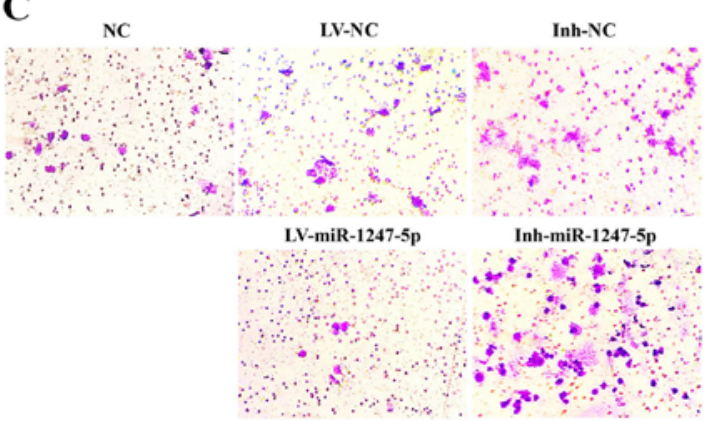

B

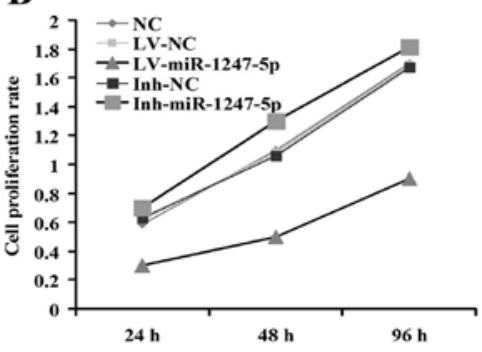

D

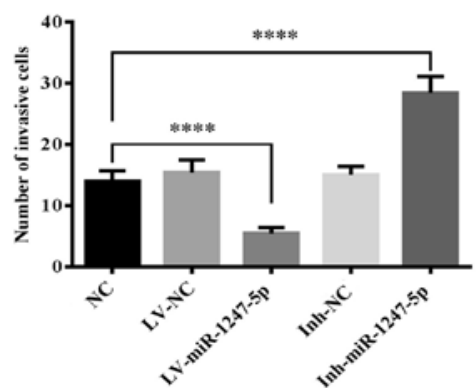

Figure 2. miR-1247-5p inhibits the proliferation and invasion in vitro. HepG2 (NC) and HepG2 cells infected with lentiviral-mediated miR-1247-5p (LV-miR1247-5p) or its negative control (LV-NC), and HepG2 cells transfected with the inhibitor of miR-1247-5p (Inh-miR-1247-5p) or its negative control (Ihn-NC) were used in the following experiments. (A) The mRNA expression of miR-1247-5p in the HepG2 cells of each group. Graphs represent the relative expression values, normalized against the reference gene. (B) The cell proliferation rate of each group was detected after 24,48 or 72 h of incubation using the CCK- 8 assay. (C) The invasive activity was detected using Transwell assay. The cells were stained with $0.1 \%$ crystal violet, photographed under a bright field (magnification, x100), and (D) the number of cells in each group was calculated and analyzed using GraphPad Prism 6 software compared with that in the control group. Data are expressed as the mean $\pm \mathrm{SD} ;{ }^{* * * *} \mathrm{p}<0.0005,{ }^{* * * *} \mathrm{p}<0.0001$.

negative control (LV-NC), and HepG2 cells transfected with the inhibitor of miR-1247-5p (Inh-miR-1247-5p) or its negative control (Ihn-NC) were detected by qRT-PCR. Normal HepG2 cells were used as the normal control (NC). The results revealed that the mRNA expression of miR-1247-5p was 8.9 times higher in the LV-miR-1247-5p-infected group and 5.4 times lower in the Inh-miR1247-5p-transfected group compared to that of the NC group $(\mathrm{p}<0.0005)$ (Fig. 2A). These results indicated that the cell models of miR-1247-5p were successfully established. Then, the CCK-8 assay was performed to explore the role of miR-1247-5p in cell proliferation and the results revealed that the ectopic overexpression of miR-1247-5p significantly decreased the proliferative rate of HepG2 cells, whereas the decreased expression of miR-1247-5p induced the enhancement of cell proliferation (Fig. 2B). Then, the role of miR-1247-5p in cell invasion was assessed using the Transwell assay. The results revealed that the invasive activity in the LV-miR-1247-5p-infected group was significantly decreased $(\mathrm{p}<0.0001)$, whereas that in the Inh-miR-1247-5p-transfected goup was significantly enhanced $(\mathrm{p}<0.0001)$ compared to that of the control groups (Fig. 2C and D). These results demonstrated that the ectopic overexpression of miR-1247-5p inhibited the proliferation and invasion of HepG2 cells in vitro.

miR-1247-5p induces cell apoptosis in vitro. The role of miR-1247-5p in the regulation of the cell cycle was analyzed using flow cytometry. The results revealed that the number of apoptotic cells or cell debris increased significantly in the LV-miR-1247-5p-infected group $(\mathrm{p}<0.05)$. In contrast, that in the Inh-miR-1247-5p transfected group was slightly decreased compared to that in the control group, and there was no difference in the proportion of HepG2 cells in the G0/G1, G2/M and $\mathrm{S}$ phases between the different groups (Fig. 3A and B). To further demonstrate this, Hoechst staining assay was performed. The results revealed that an increased abundance of cells with characteristics of chromosome condensation was found in the cells infected with the LV-miR-1247-5p (Fig. 3C). These results demonstrated that the ectopic overexpression of miR-1247-5p induced the apoptosis of HepG2 cells in vitro.

miR-1247-5p inhibits the growth of tumors in vivo. To demonstrate that miR-1247-5p can inhibit the growth of tumors in vivo, a nude mouse tumorigenicity assay was performed. HepG2 cells infected with lentiviral-mediated miR-1247-5p (LV-miR-1247-5p) or negative control vectors ( $\mathrm{LV}-\mathrm{NC}$ ) were subcutaneously injected at the lateral axillary of BALB/c nude mice. The tumor size of each mouse was assessed once every two days for four weeks. Subsequently, the experimental mice were sacrificed and the tumors were photographed. The results revealed that the tumor size of the experimental mice was significantly smaller than that of the control group of mice (Fig. 4A and B). These results demonstrated that the ectopic overexpression of miR-1247-5p inhibited the growth of HCC tumors in vivo. Incidentally, during this experiment, one mouse in the experimental group died for unknown reasons, and one mouse in the control group did not form tumors.

Wnt3 is a target of miR-1247-5p in HepG2 cells. It is generally understood that miRNAs execute post-transcriptional regulation by binding to the 3 'UTR of their downstream 
A
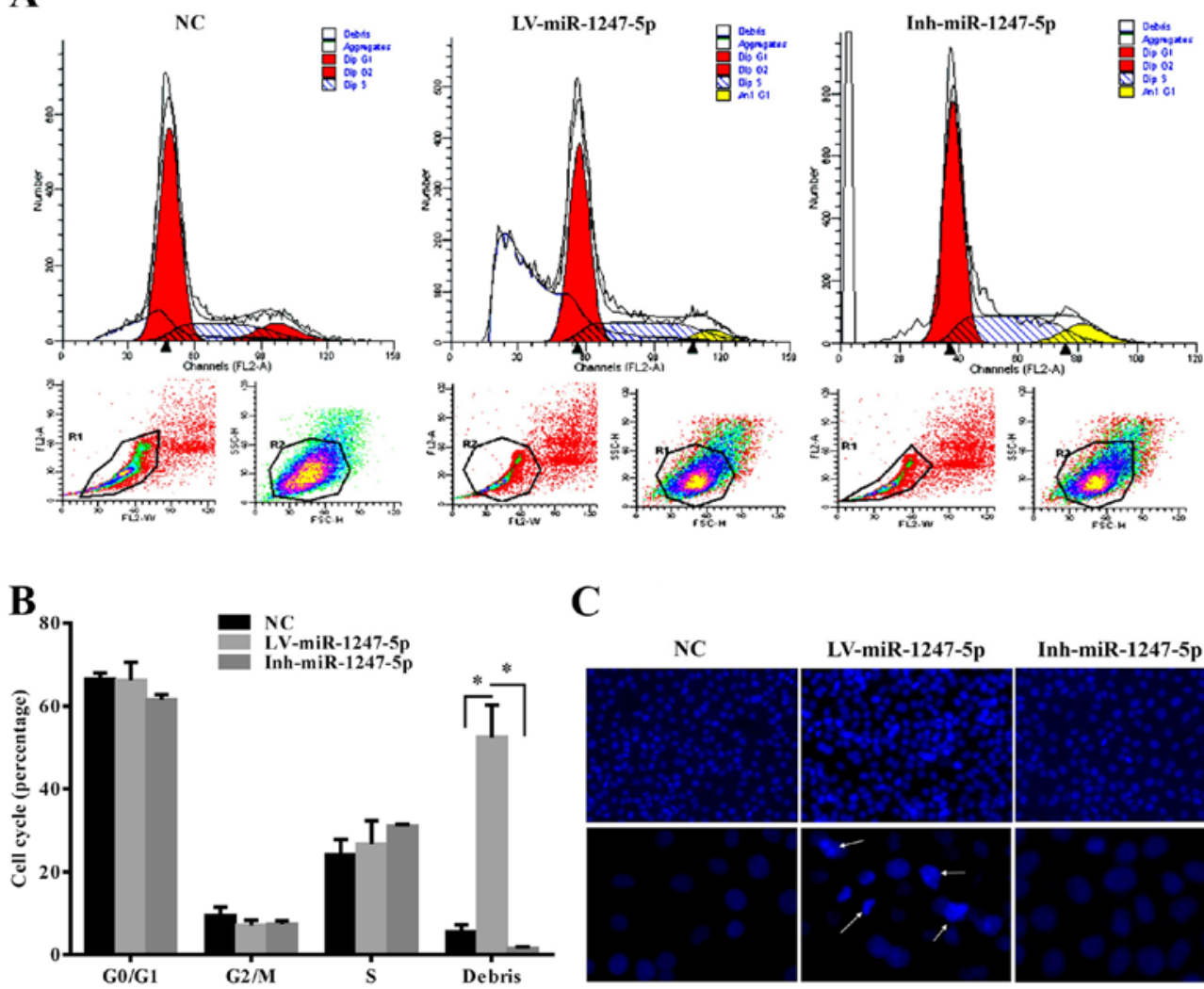

C
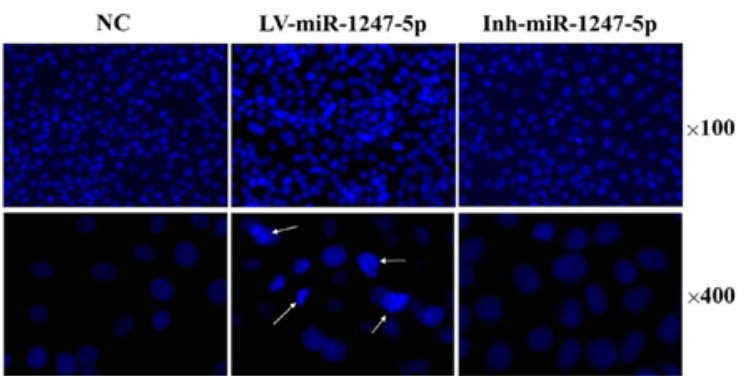

Figure 3. miR-1247-5p induces cell apoptosis in vitro. (A and B) Flow cytometric analysis revealed an induction of the apoptotic cell fraction in the LV-miR-1247-5p-infected cells at $48 \mathrm{~h}$ post-infection. Hoechst staining revealed the morphology of nuclei in the HepG2 cells and the cells transfected with Inh-miR1247-5p, abundant nuclei with chromosome condensation were observed in cells infected with LV-miR-1247-5p (C, arrows) at $48 \mathrm{~h}$ post-infection The data in B represent the mean $\pm \mathrm{SD} ;{ }^{*} \mathrm{p}<0.05$.

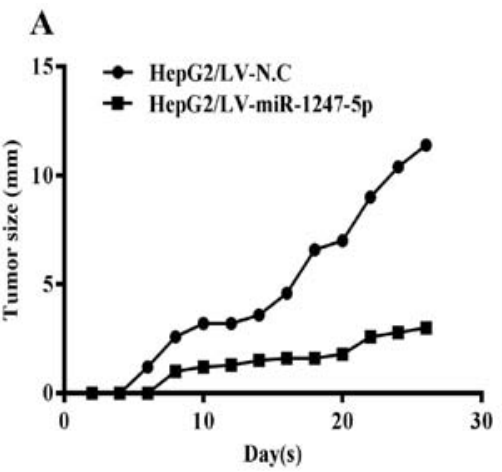

B

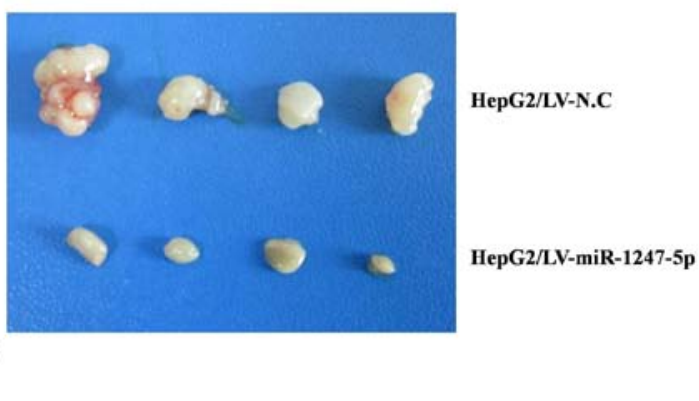

Figure 4. miR-1247-5p inhibits the tumor growth in vivo. (A) The size of the tumors in mice subcutaneously injected with HepG2 cells infected with LV-miR1247-5p (HepG2/miR-1247-5p) or lentivirus empty plasmid (HepG2/LV-NC). The size of the tumors was assessed once every two days for 4 weeks. (B) The appearance of the tumors in the miR-1247-5p overexpressing mice and the control mice.

genes. To explore the molecular mechanisms involved in the miR-1247-5p-mediated tumor suppression, target genes of miR-1247-5p were analyzed using the online target prediction software DIANA-MICROT (http://diana. imis.athena-innovation.gr) and miRanda (www. micorrna. org), and the correlative signaling pathway regulated by miR-1247-5p was analyzed by CytoScape 3.0 software. The results revealed that Wnt/ $\beta$-catenin and MAPK were the two most related pathways, and wingless-type MMTV integration site family, member 3 (Wnt3), a potential target gene of miR-1247-5p, was chosen as the focus of our research in the present study based on the complementarity of its sequence to miR-1247-5p and the important role of the Wnt/ $/$-catenin pathway in HCC. In contrast to the sequence of miR-1247-5p, there were seven consecutive binding sites in the 3'UTR of wild-type Wnt3 (Wnt3 3'UTR) and four consecutive binding sites in mutant Wnt3 3'UTR (mut-Wnt3 3'UTR) (Fig. 5A). To further confirm that there was a direct interaction between miR-1247-5p and Wnt3, a luciferase reporter assay was performed in HepG2 cells co-transfected with the luciferase reporter vector expressing the 3'UTR of Wnt3 and LV-miR1247-5p, Inh-miR-1247-5p or negative control vectors. Vectors 
$\mathbf{A}$

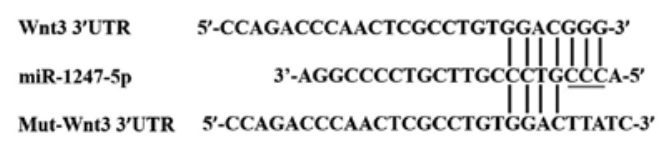

C

NC

LV-NC

LV-miR-1247-5p

Inh-NC

Inh-miR-1247-5p

GAPDH
B

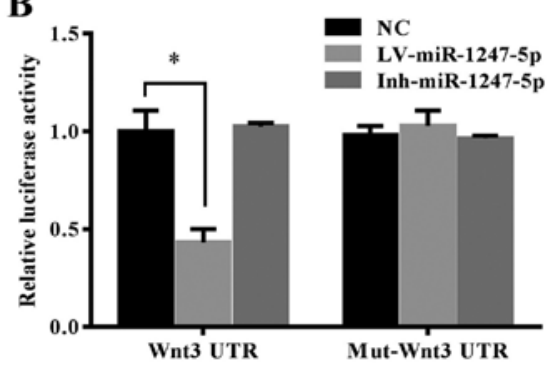

D

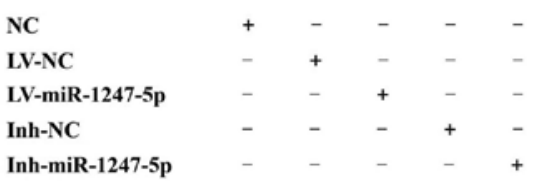

$\beta$-catenin

$\beta$-actin

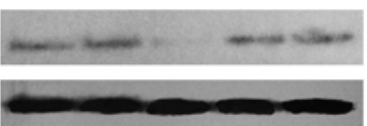

Figure 5. miR-1247-5p targets the Wnt3 gene. (A) Sequence of potential binding sites of miR-1247-5p in the 3'UTR of Wnt3 mRNA (top line). Mutations were introduced into the binding sites for generation of mutated Wnt3 3'TUR (bottom line). (B) Validation of the miR-1247-5p target using Wnt3 3'UTR luciferase reporter vector. The expression of (C) Wnt3 and its downstream protein (D) $\beta$-catenin was detected by immunoblotting in the HepG2 cells transfected with the indicated plasmids at $48 \mathrm{~h}$ post-transfection. The results represent the mean $\pm \mathrm{SD}$; ${ }^{*} \mathrm{p}<0.05$. Wnt3, wingless-type MMTVintegration site family, member 3 .

A

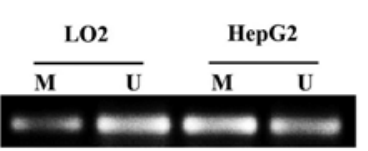

C

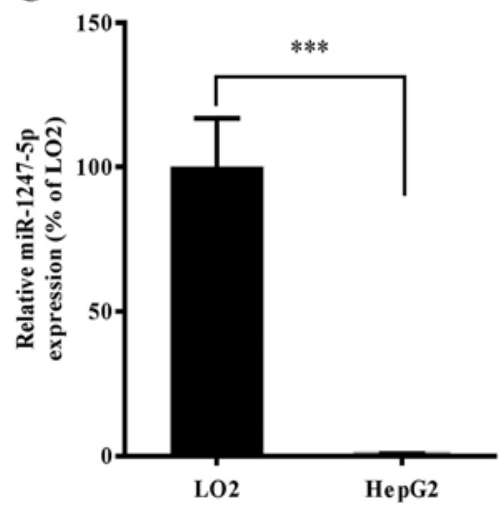

B

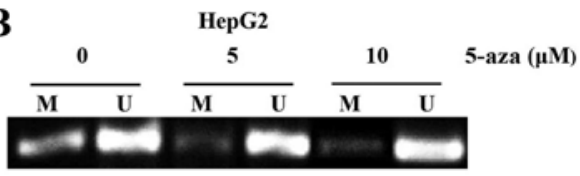

D

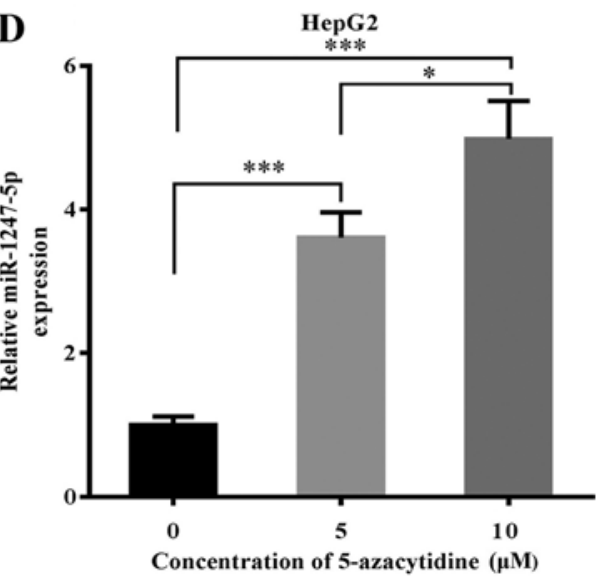

Figure 6. DNA methylation regulates the expression of miR-1247-5p. (A and C) Methylation level and mRNA expression of miR-1247-5p in HepG2 and LO2 cells, and (B and D) HepG2 cells treated with 5 and $10 \mu \mathrm{mol} / 1$ demethylation drug 5-azacytidine (5-Aza) were assessed by DNA methylation-specific PCR (MSP) and qRT-PCR. M, represents methylated PCR products and U, represents unmethylated PCR products. Graphs in (C and D) represent the relative expression values of miR-1247-5p normalized to that of normal LO2 or HepG2 cells. The results represent the mean $\pm \mathrm{SD} ;{ }^{*} \mathrm{p}<0.05,{ }^{* * * *} \mathrm{p}<0.0005$.

expressing the mutant 3 'UTR of the Wnt3 gene were used as the control. A significant decrease in the luciferase signal was observed in cells co-transfected with the Wnt3 3'UTR vector and the LV-miR-1247-5p. In contrast, the inhibition was fully rescued when target sites were mutated (Fig. 5B). These results revealed that miR-1247-5p could inhibit the transcription activity of the Wnt3 gene by targeting its 3'UTR. To further demonstrate the inhibitory effect of miR-1247-5p on the expression of Wnt3, the expression of Wnt 3 and its downstream protein $\beta$-catenin in each group was analyzed by western blotting. The results revealed that $\mathrm{Wnt} 3$ and $\beta$-catenin were significantly decreased at the protein level after ectopic overexpression of miR-1247-5p. In contrast, the protein expression of Wnt 3 and $\beta$-catenin was slightly increased in the Inh-miR-1247-5p transfected group (Fig. 5C and D).

DNA methylation regulates the expression of miR-1247-5p. Based on the study of Anwar et al that revealed that the miR-1247-5p gene was hypermethylated in HCC, we surmised that DNA methylation may play an important role in the regulation of the expression of miR-1247-5p. To further explore the role of DNA methylation in the regulation of miR-1247-5p 
expression in HCC, the sequence of the miR-1247-5p gene was analyzed using UCSC Genome Database (http://genome. ucsc.edu/). The results revealed that the promoter region of the miR-1247-5p gene was 5,578 bp in length, located on chromosome 14 between $101,559,653$ up to $101,565,230 \mathrm{bp}$. In addition, the CG content of this region accounted for $62.4 \%$, and the possibility of a $\mathrm{CpG}$ island existing in this region was 0.84 (data not shown). These results revealed that a $\mathrm{CpG}$ island most probably existed in the promoter region of the miR-1247-5p gene. To confirm that the expression of miR-1247-5p was regulated by DNA methylation, the methylation level of the miR-1247-5p gene in HepG2 cells (an HCC cell line) and LO2 cells (a normal liver cell line) was detected using MSP assay and the expression of miR-1247-5p in HepG2 cells was assessed by qRT-PCR after treatment with demethylating drug, 5-azacytidine. The results revealed that the methylation level of miR-1247-5p increased significantly and the expression of miR-1247-5p decreased significantly in the HepG2 cells compared to that of LO2 cells (Fig. 6A and C). Following treatment with 5-azacytidine, the methylation level of miR-1247-5p in HepG2 cells decreased and the expression of miR-1247-5p in these cells significantly increased (Fig. 6B and D). Based on these results, we demonstrated that the expression of miR-1247-5p could be regulated by DNA methylation.

\section{Discussion}

Dysregulation of miRNAs has been demonstrated to be involved in tumorigenesis and progression in various types of tumors, and the role of miRNAs in the development of cancers has gradually been recognized (20-22). However, the elucidation of the role of miRNAs in HCC is still in the early developmental stage. miR-1247-5p, transcripted by DLKI-DIO3 domain, is located at the distal end of human chromosome 14 and conservatively expressed at a high level in mammalian species. Previous studies have reported that miR$1247-5 p$ is differentially expressed in various types of cancer, and had positive or negative effects on the proliferation, invasion and apoptosis of different cancer cells (15-18). However, the role of miR-1247-5p in HCC is largely unknown.

In the present study, it was demonstrated that miR-1247-5p was significantly downregulated in serum and tumor samples of patients with HCC and in HCC cell lines. miRNAs existing in the sera are usually called circulating miRNAs. Circulating miRNAs have tumor-marker characteristics, and tumors at different stages of development have different miRNA expression profiles (23). A recent study revealed that circulating miRNAs may be derived from active secretion of tissue cells (24). It has been reported that circulating miRNAs could be used in the early diagnosis of many cancers, such as gastric (25), prostatic (26), esophageal (27), bladder (28), ovarian (29) and nasopharyngeal cancer (30). Due to the existence of miR-1247-5p in sera, it is suggested that the detection of the expression of miR-1247-5p in sera can be used as a non-invasive approach for the early diagnosis of HCC. In a recent study, researchers found that miR1247-5p was downregulated in pancreatic cancer, and could inhibit cancer cell proliferation by targeting neuropilins (17). Thus, it was determined that miR-1247-5p is a tumor-suppressor miRNA in pancreatic cancer. However, another research group found a significant upregulation of miR-1247-5p in castration- resistant prostate cancer (CRPC) samples and prostate cancer (PC) cell lines, therefore miR-1247-5p functioned as a oncomiRNA (18). In the present study, miR-1247-5p was significantly downregulated in clinical samples of patients with $\mathrm{HCC}$ and HCC cell lines. Ectopic overexpression of miR-1247-5p significantly inhibited the proliferation and invasion of HepG2 cells, induced cell apoptosis, and suppressed tumor growth in vivo via the regulation of the expression of $\mathrm{Wnt} 3$. These results indicated that miR-1247-5p functioned as a tumor suppressor in HCC. We suggest that the different effects of miR-1247-5p on cell proliferation and invasion of cancer cells depended on the type of cancer or the genes it was targeting.

To explore the regulatory mechanisms involved in miR-1247-5p-mediated tumor suppression, putative target genes of miR-1247-5p were analyzed by prediction software. Wingless-type MMTV integration site family, member 3 (Wnt3), a secreted glycoprotein, located on human chromosome 17 and an upstream protein of the canonical $\mathrm{Wnt} / \beta$-catenin pathway (31), was predicted as a potential target gene of miR$1247-5 p$ and this was ascertained by luciferase reporter assay and western blotting. Previous studies demonstrated that the canonical $\mathrm{Wnt} / \beta$-catenin pathway participates in the genesis and progression of several cancers by activating the transcription of its downstream genes (32-34). In brief, when stimulated by signals, Wnt proteins bind with transmembrane receptors to activate the expression of dishevelled proteins (DVL), which inhibit the activity of GSK3 $\beta$ in the degradation complex, preventing the phosphorylation of $\beta$-catenin by GSK3 $\beta$, thus avoiding its identification and degradation from ubiquitin or proteasomes. Then, $\beta$-catenin accumulates in the cytoplasm and translocates to the nucleus, where it binds with nuclear transcription factors and activates the expression of downstream target genes, which leads to the abnormal proliferation of cells and the occurrence of tumors. Our results indicated that the ectopic overexpression of miR-1247-5p inhibited the expression of the Wnt3 protein in HepG2 cells by targeting the 3'UTR of the Wnt 3 gene, and $\beta$-catenin, a downstream protein of the Wnt/ $\beta$-catenin pathway could also be inhibited. These results demonstrated the role of miR-1247-5p in the inhibition of the proliferation and invasion of HCC cells partially achieved via the regulation of the activity of the $\mathrm{Wnt} / \beta$-catenin pathway.

DNA methylation in miRNA genes could serve as a new biomarker for the early detection, diagnosis and prognosis of malignant tumors. Generally, miRNAs, which function as tumor suppressors, are silenced by DNA hypermethylation, which results in the overexpression of downstream oncogenes and the occurrence of tumors $(35,36)$. However, knowledge concerning microRNA gene methylation in human HCC is still limited. To explore the role of DNA methylation in the regulation of miR-1247-5p expression in HCC, the UCSC genome database (http:/genome.ucsc.edu/) was used to analyze the promoter region of the hsa-miR-1247-5p gene, and the methylation level of the miR-1247-5p gene in HepG2 cells and normal liver cells which was detected by MSP assay. The results revealed that a $\mathrm{CpG}$ island most probably exists in the promoter region of the miR-1247-5p gene. The methylation level of the miR-1247-5p gene was significantly increased in HepG2 cells compared to that of normal liver cells. Following treatment with demethylation drug 5-azacytidine, the methylation level of the miR-1247-5p gene was decreased and the expression of miR-1247-5p was 
significantly increased. These results reveal that the expression of miR-1247-5p could be regulated by DNA methylation.

In summary, these results demonstrated that miR-1247-5p expression is downregulated in HCC tumors, and influence the proliferation and invasion of HepG 2 cells via the regulation of the Wnt/ $\beta$-catenin pathway, and can be regulated by DNA methylation. These data reveal that miR-1247-5p functions as a tumor suppressor in $\mathrm{HCC}$, and can be used as a new biomarker and potential target for the diagnosis and treatment of HCC. Further studies should focus on the regulatory mechanism of miR-1247-5p in the genesis and progression of HCC.

\section{Acknowledgements}

The present study was supported by grants from the Natural Science Foundation of China (no. 81460368), the Ningxia High Education Science and Technology Important Project [(2014) no. 2014-70], and the Science and Technology Program of Ningxia (2013).

\section{References}

1. Ferlay J, Shin HR, Bray F, Forman D, Mathers C and Parkin DM: Estimates of worldwide burden of cancer in 2008: GLOBOCAN 2008. Int J Cancer 127: 2893-2917, 2010.

2. Lee S, Lee HJ, Kim JH, Lee HS, Jang JJ and Kang GH: Aberrant $\mathrm{CpG}$ island hypermethylation along multistep hepatocarcinogenesis. Am J Pathol 163: 1371-1378, 2003.

3. Søkilde R, Vincent M, Møller AK, Hansen A, Høiby PE, Blondal T, Nielsen BS, Daugaard G, Møller S and Litman T: Efficient identification of miRNAs for classification of tumor origin. J Mol Diagn 16: 106-115, 2014.

4. Osman A: MicroRNAs in health and disease - basic science and clinical applications. Clin Lab 58: 393-402, 2012.

5. Zhao G, Cai C, Yang T, Qiu X, Liao B, Li W, Ji Z, Zhao J, Zhao H, Guo M, et al: MicroRNA-221 induces cell survival and cisplatin resistance through PI3K/Akt pathway in human osteosarcoma. PLoS One 8: e53906, 2013.

6. Mendell JT and Olson EN: MicroRNAs in stress signaling and human disease. Cell 148: 1172-1187, 2012.

7. Lewis BP, Burge CB and Bartel DP: Conserved seed pairing, often flanked by adenosines, indicates that thousands of human genes are microRNA targets. Cell 120: 15-20, 2005.

8. Hayes J, Peruzzi PP and Lawler S: MicroRNAs in cancer: Biomarkers, functions and therapy. Trends Mol Med 20: 460-469, 2014.

9. van Rooij E and Kauppinen S: Development of microRNA therapeutics is coming of age. EMBO Mol Med 6: 851-864, 2014.

10. Nugent M: MicroRNA function and dysregulation in bone tumors: The evidence to date. Cancer Manag Res 6: 15-25, 2014.

11. Liu AM, Poon RT and Luk JM: MicroRNA-375 targets Hipposignaling effector YAP in liver cancer and inhibits tumor properties. Biochem Biophys Res Commun 394: 623-627, 2010.

12. Fornari F, Milazzo M, Chieco P, Negrini M, Calin GA, Grazi GL, Pollutri D, Croce CM, Bolondi L and Gramantieri L: MiR-199a-3p regulates mTOR and c-Met to influence the doxorubicin sensitivity of human hepatocarcinoma cells. Cancer Res 70: 5184-5193, 2010.

13. Morin RD, O'Connor MD, Griffith M, Kuchenbauer F, Delaney A, Prabhu AL, Zhao Y, McDonald H, Zeng T, Hirst M, et al: Application of massively parallel sequencing to microRNA profiling and discovery in human embryonic stem cells. Genome Res 18: 610-621, 2008.

14. Martinez-Sanchez A and Murphy CL: miR-1247 functions by targeting cartilage transcription factor SOX9. J Biol Chem 288: 30802-30814, 2013.

15. Wu Y, Ginther C, Kim J, Mosher N, Chung S, Slamon D and Vadgama JV: Expression of Wnt 3 activates Wnt $\beta$-catenin pathway and promotes EMT-like phenotype in trastuzumabresistant HER2-overexpressing breast cancer cells. Mol Cancer Res 10: 1597-1606, 2012.
16. Yan $\mathrm{H}$, Choi AJ, Lee $\mathrm{BH}$ and Ting $\mathrm{AH}$ : Identification and functional analysis of epigenetically silenced microRNAs in colorectal cancer cells. PLoS One 6: e20628, 2011.

17. Shi S, Lu Y, Qin Y, Li W, Cheng H, Xu Y, Xu J, Long J, Liu L, Liu C, et al: miR-1247 is correlated with prognosis of pancreatic cancer and inhibits cell proliferation by targeting neuropilins. Curr Mol Med 14: 316-327, 2014

18. Scaravilli M, Porkka KP, Brofeldt A, Annala M, Tammela TL, Jenster GW, Nykter M and Visakorpi T: MiR-1247-5p is overexpressed in castration resistant prostate cancer and targets MYCBP2. Prostate 75: 798-805, 2015.

19. Anwar SL, Albat C, Krech T, Hasemeier B, Schipper E, Schweitzer N, Vogel A, Kreipe H and Lehmann U: Concordant hypermethylation of intergenic microRNA genes in human hepatocellular carcinoma as new diagnostic and prognostic marker. Int J Cancer 133: 660-670, 2013.

20. Han G, Wang Y, Bi W, Jia J and Wang W: MicroRNA-124 functions as a tumor suppressor and indicates prognosis in human osteosarcoma. Exp Ther Med 9: 679-684, 2015.

21. Mardin WA and Mees ST: MicroRNAs: Novel diagnostic and therapeutic tools for pancreatic ductal adenocarcinoma? Ann Surg Oncol 16: 3183-3189, 2009.

22. Rachagani S, Kumar S and Batra SK: MicroRNA in pancreatic cancer: Pathological, diagnostic and therapeutic implications. Cancer Lett 292: 8-16, 2010.

23. Bianchi F, Nicassio F, Marzi M, Belloni E, Dall'olio V, Bernard L, Pelosi G, Maisonneuve P, Veronesi G and Di Fiore PP: A serum circulating miRNA diagnostic test to identify asymptomatic high-risk individuals with early stage lung cancer. EMBO Mol Med 3: 495-503, 2011.

24. Valadi H, Ekström K, Bossios A, Sjöstrand M, Lee JJ and Lötvall JO: Exosome-mediated transfer of mRNAs and microRNAs is a novel mechanism of genetic exchange between cells. Nat Cell Biol 9: 654-659, 2007.

25. Liu R, Zhang C, Hu Z, Li G, Wang C, Yang C, Huang D, Chen X, Zhang H, Zhuang R, et al: A five-microRNA signature identified from genome-wide serum microRNA expression profiling serves as a fingerprint for gastric cancer diagnosis. Eur J Cancer 47: 784-791, 2011.

26. Chen ZH, Zhang GL, Li HR, Luo JD, Li ZX, Chen GM and Yang J: A panel of five circulating microRNAs as potential biomarkers for prostate cancer. Prostate 72: 1443-1452, 2012.

27. Zhang C, Wang C, Chen X, Yang C, Li K, Wang J, Dai J, Hu Z, Zhou X, Chen L, et al: Expression profile of microRNAs in serum: A fingerprint for esophageal squamous cell carcinoma. Clin Chem 56: 1871-1879, 2010.

28. Long JD, Sullivan TB, Humphrey J, Logvinenko T, Summerhayes KA, Kozinn S, Harty N, Summerhayes IC, Libertino JA, Holway AH, et al: A non-invasive miRNA based assay to detect bladder cancer in cell-free urine. Am J Transl Res 7: 2500-2509, 2015

29. Suryawanshi S, Vlad AM, Lin HM, Mantia-Smaldone G, Laskey R, Lee M, Lin Y, Donnellan N, Klein-Patel M, Lee T, et al: Plasma microRNAs as novel biomarkers for endometriosis and endometriosis-associated ovarian cancer. Clin Cancer Res 19: 1213-1224, 2013.

30. Zeng X, Xiang J, Wu M, Xiong W, Tang H, Deng M, Li X, Liao Q, Su B, Luo Z, et al: Circulating miR-17, miR-20a, miR-29c, and miR-223 combined as non-invasive biomarkers in nasopharyngeal carcinoma. PLoS One 7: e46367, 2012.

31. Nakashima N, Liu D, Huang CL, Ueno $M$, Zhang $X$ and Yokomise $\mathrm{H}$ : Wnt 3 gene expression promotes tumor progression in non-small cell lung cancer. Lung Cancer 76: 228-234, 2012.

32. White BD, Chien AJ and Dawson DW: Dysregulation of Wnt $/ \beta$ catenin signaling in gastrointestinal cancers. Gastroenterology 142 : 219-232, 2012.

33. Lachenmayer A, Alsinet C, Savic R, Cabellos L, Toffanin S, Hoshida Y, Villanueva A, Minguez B, Newell P, Tsai HW, et al: Wnt-pathway activation in two molecular classes of hepatocellular carcinoma and experimental modulation by sorafenib. Clin Cancer Res 18: 4997-5007, 2012.

34. Voloshanenko O, Erdmann G, Dubash TD, Augustin I, Metzig M, Moffa G, Hundsrucker C, Kerr G, Sandmann T, Anchang B, et al: Wnt secretion is required to maintain high levels of Wnt activity in colon cancer cells. Nat Commun 4: 2610, 2013.

35. Kunej T, Godnic I, Ferdin J, Horvat S, Dove P and Calin GA: Epigenetic regulation of microRNAs in cancer: An integrated review of literature. Mutat Res 717: 77-84, 2011.

36. Kozaki K and Inazawa J: Tumor-suppressive microRNA silenced by tumor-specific DNA hypermethylation in cancer cells. Cancer Sci 103: 837-845, 2012. 